Supporting Information for

\title{
Disaggregation behavior of amyloid $\beta$ fibrils by anthocyanins studied by total-internal-reflection-fluorescence microscopy coupled with wireless quartz-crystal microbalance biosensor
}

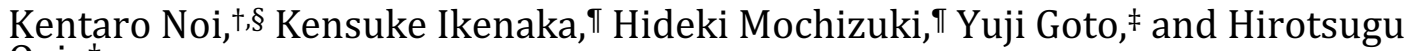
Ogi*†

†Graduate School of Engineering, Osaka University, Suita, Osaka 565-0871, Japan $\ddagger$ Global Center for Medical Engineering and Informatics, Osaka University, Suita, Osaka 565-0871, Japan

IDepartment of Neurology, Osaka University Graduate School of Medicine, 2-2 Yamadaoka, Suita, Osaka 565-0871, Japan

$\S$ Present address: Institute for NanoScience Design, Osaka University, Toyonaka, Osaka 560-8531, Japan

*E-mail: ogi@prec.eng.osaka-u.ac.jp

Table of Content

Supplementary Figure S1 … Page S2

Supplementary Figure S2 $\cdots$ Page S3

Supplementary Figure S3 $\cdots$ Page S4

Supplementary Figure S4 $\cdots$. Page S5 

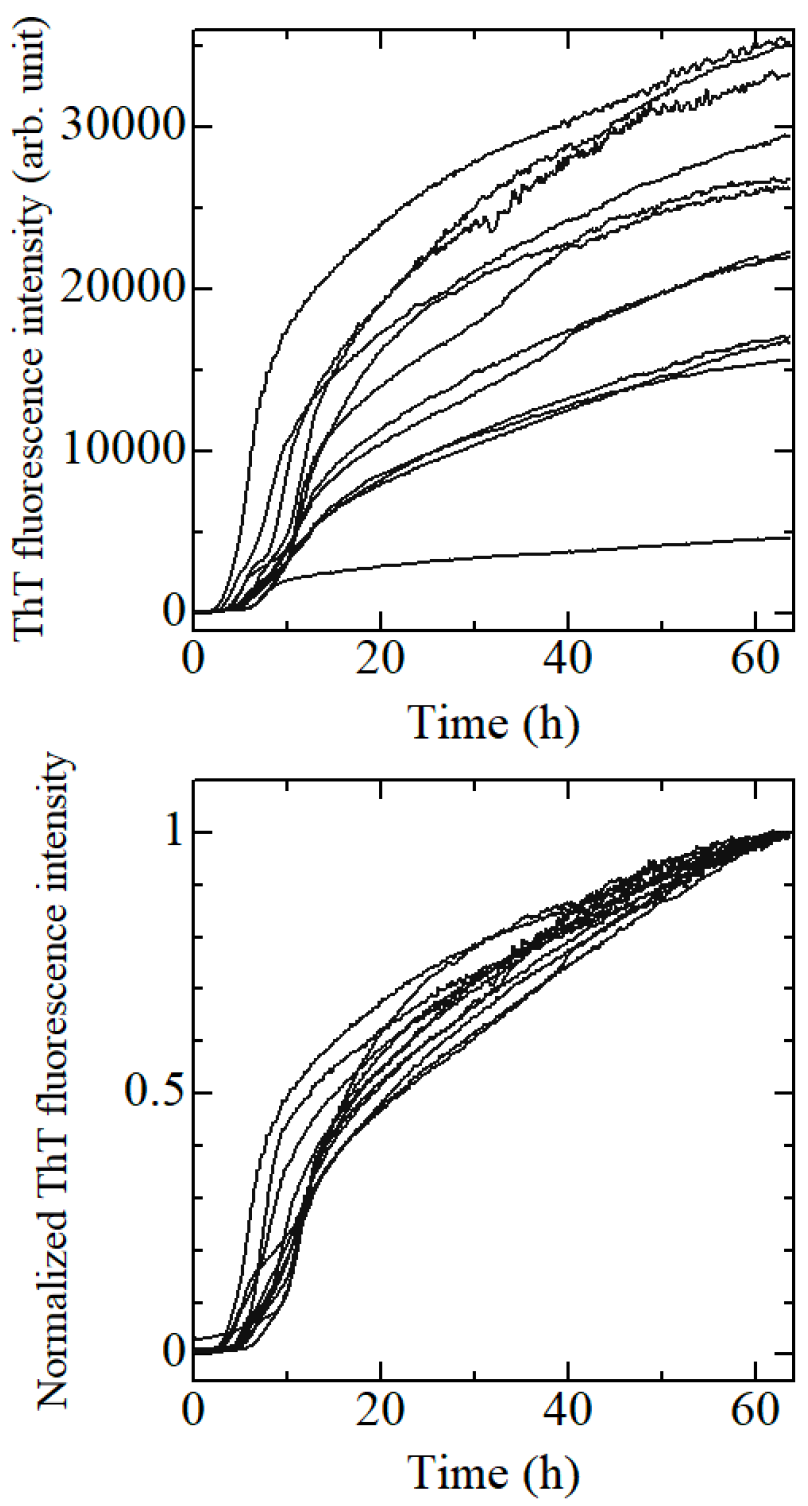

Figure S1 ThT fluorescence assay during the $A \beta$-amyloid-fibril formation. The $\mathrm{A} \beta$ amyloid fibrils were formed by adding $A \beta_{1-40}$ peptides to the $A \beta_{1-42}$ seeds inside the wells. Upper figure shows as measured ThT-fluorescence intensity for 12 wells, and the lower one shows their normalized behavior, which are nearly identical to each other. 

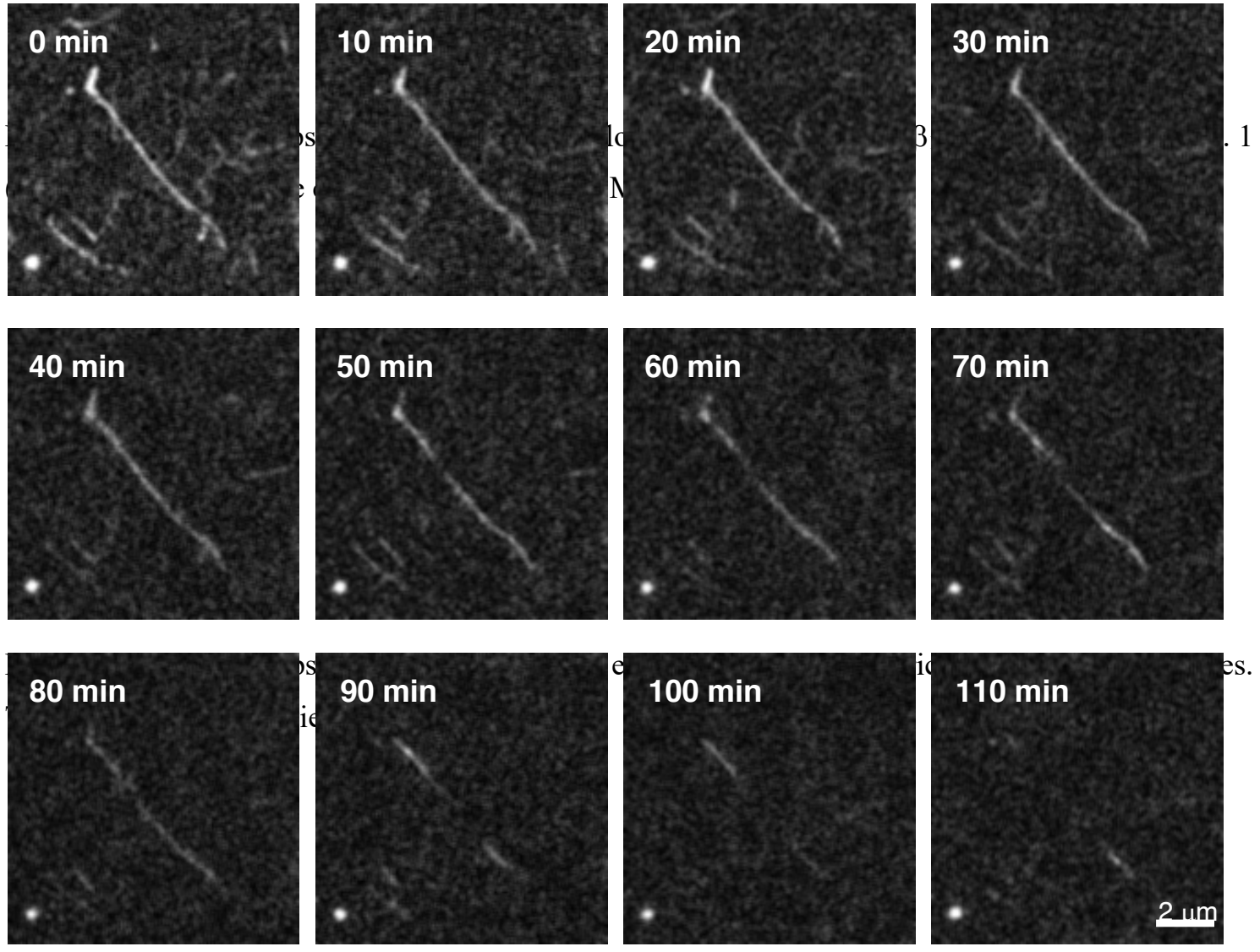

Figure S2 Disaggregation behavior of an amyloid fibril caused by D3-gal. The fibril disaggregation occurs from side walls, not from the edges. 

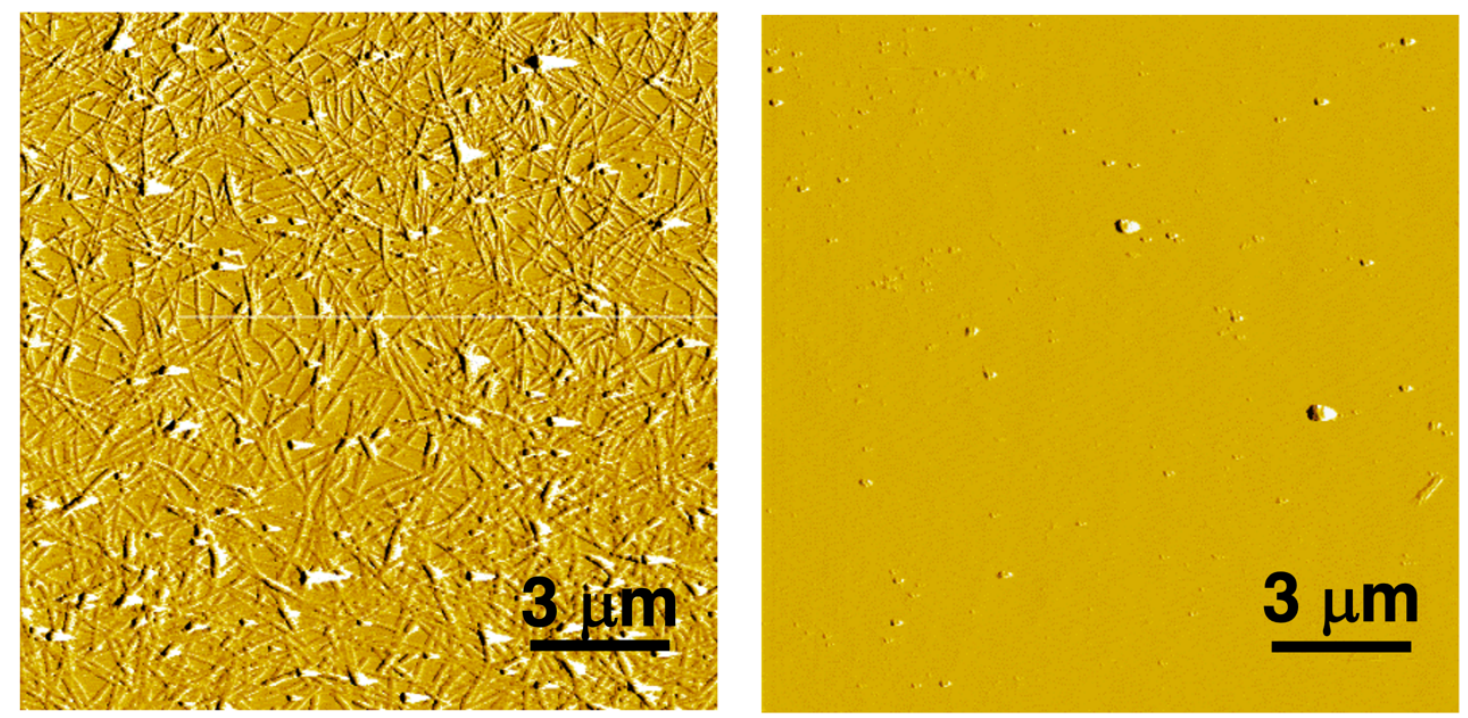

Figure S3 AFM images before injecting the D3-gal solution (left) and after flowing the D3-gal solution (right). 
a
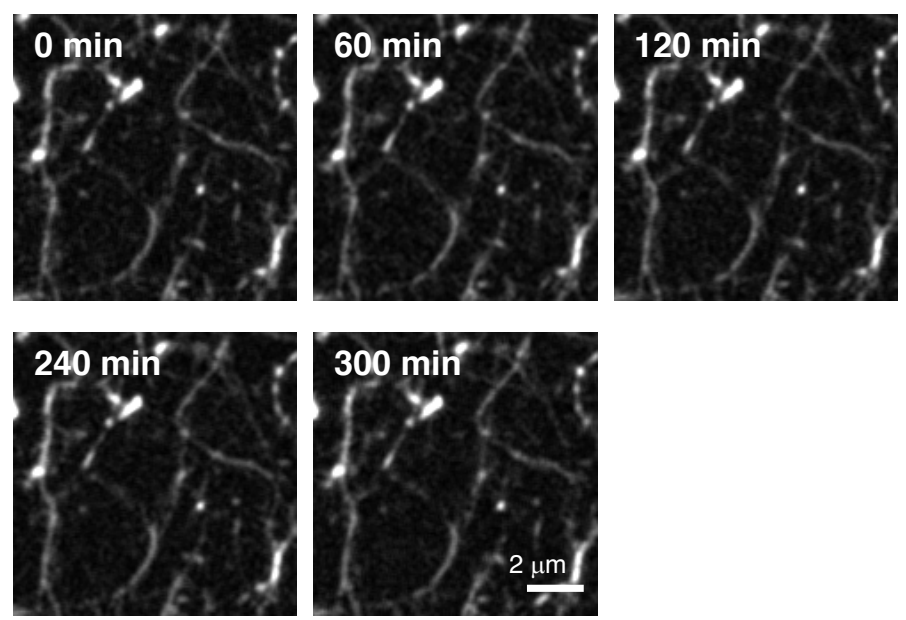

b
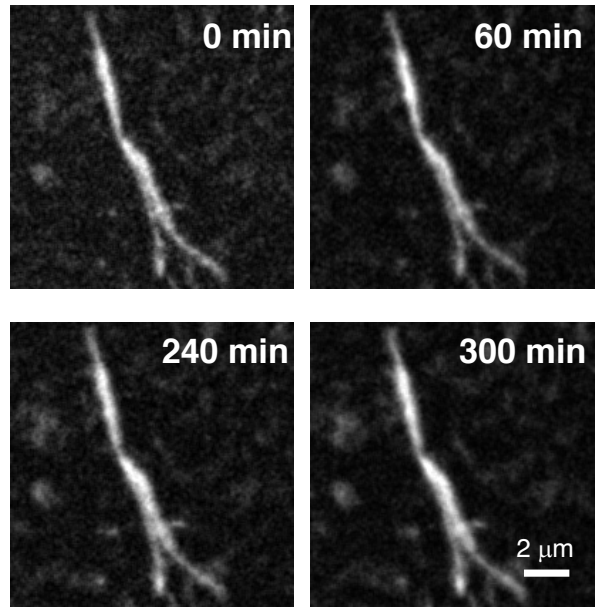

C
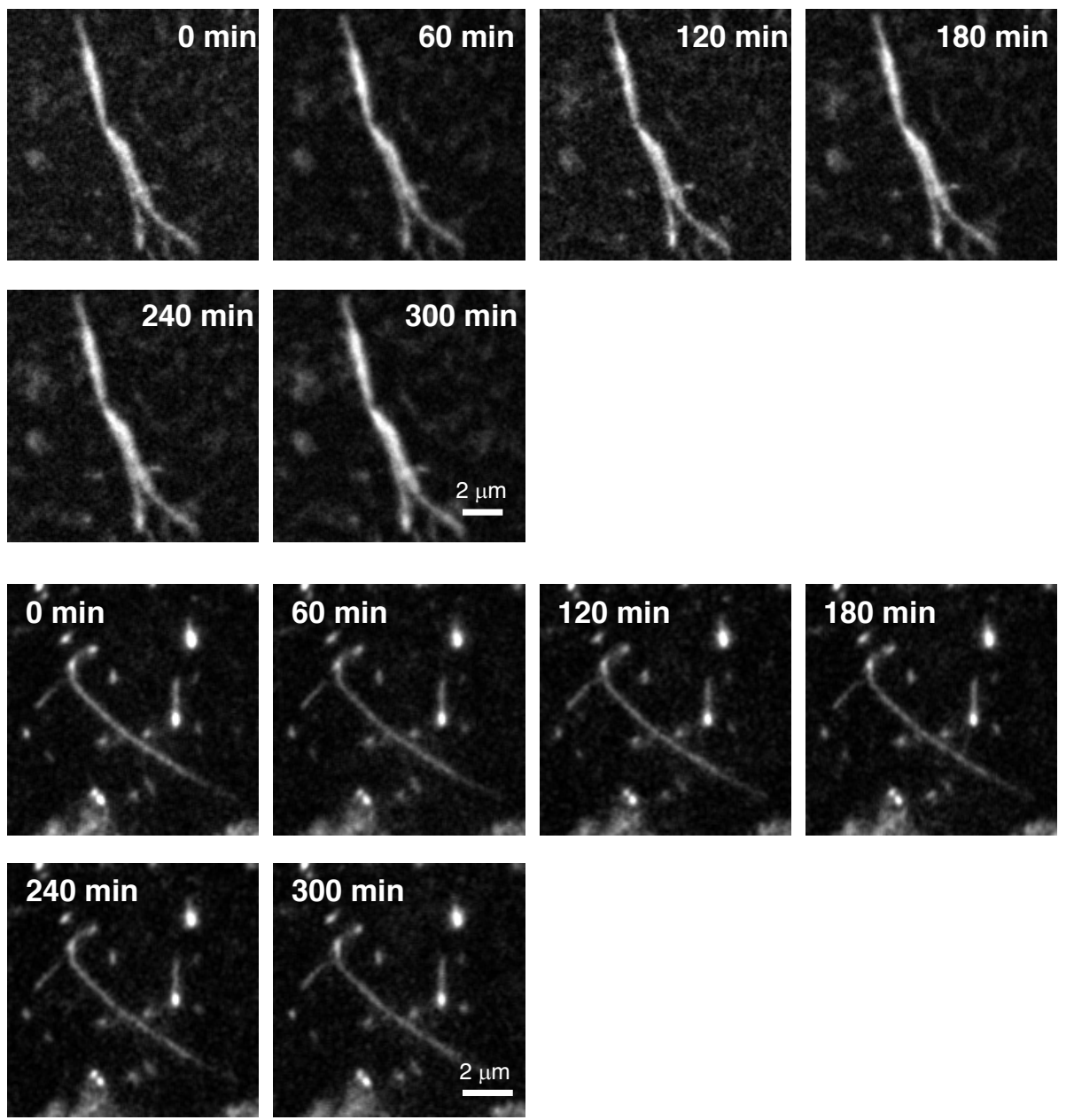

Figure S4 Amyloid fibrils observed during flows of (a) C3-gal, (b) M3-gal, and (c) CGA solutions. Unlike D3-gas, these polyphenols fail to show the disassembly activity to the fibrils. 